\title{
Extrasynaptic neurotransmission as a way of modulating neuronal functions
}

\author{
Francisco F. De-Miguel ${ }^{1 *}$ and Kjell Fuxe ${ }^{2}$ \\ Instituto de Fisiología Celular-Neurociencias, Universidad Nacional Autónoma de México, Circuito Exterior, Ciudad Universitaria, Mexico City, México \\ 2 Division of Cellular and Molecular Neuroscience, Department of Neuroscience, Karolinska Institutet, Stockholm, Sweden \\ *Correspondence: ffernand@ifc.unam.mx
}

Extrasynaptic neurotransmission allows neuronal communication at variable distances, based on the release and diffusion of chemical substances from the soma, axon, or dendrites of neurons, or after transmitter leak-out from the synaptic cleft (De-Miguel and Trueta, 2005; Fuxe et al., 2007). Extrasynaptic neurotransmission modulates the input-output relationships of whole circuits by changing the electrical properties of neuronal populations, their chemical and electrical connectivities and also by activating the transport and release of transmitters by glial cells. The term synaptic transmission immediately refers to a confined process in which the arrival of an impulse at a presynaptic ending evokes a rapid burst of exocytosis that triggers an electrical reaction in the postsynaptic cell, within millisecond time and micrometer distance scales. By contrast, extrasynaptic exocytosis involves diverse ultrastructural and mechanistic principles that, when active, expand the timing and volume possibilities of nerve cell communication (Trueta et al., 2003, 2004; De-Miguel and Trueta, 2005; Agnati et al., 2010). Extrasynaptic sites release transmitters into the extracellular fluid in the absence of identifiable postsynaptic counterparts, producing the activation mainly of extrasynaptic receptors in neighboring neurons and/or glial cells. Although extrasynaptic exocytosis is triggered by electrical activity, it may start after a lag (asynchronous transmission) and may continue for seconds or even minutes once the depolarization has ended, thus expanding the timing of neuronal signaling.

Extrasynaptic exocytosis exists in central and peripheral neurons of vertebrates and invertebrates and involves many different types of substances including low molecular weight transmitters, such as acetylcholine, GABA, glutamate, ATP, and biogenic amines, and peptides such as substance $P$, vasopressin, oxytocin, beta-endorphin, and also proteins (De-Miguel and Trueta, 2005). Diffusible modulators such as nitric oxide (Joca et al., 2007) and endocannabinoids (Yuan and Burrell, 2010) produced by postsynaptic targets also act on neighboring groups of neurons and glial cells.

The intracellular mechanisms of extrasynaptic exocytosis vary from one neuronal compartment to another in the same neuron and also from one neuron type to another (De-Miguel and Trueta, 2005). For example, in axonal varicosities, clear vesicles may be apposed to the resting plasma membrane as in canonical presynaptic active zones or may rest at a certain distance from the plasma membrane, but these endings lack morphologically-defined postsynaptic counterparts, therefore being asynaptic and sources of extrasynaptic release. Transmitters and/or peptides may also be packed onto axonal and somatic dense core vesicles, most of which rest at a distance from the plasma membrane and in response to electrical activity move towards it and fuse, as in excitable endocrine cells. A single neuron may use several of these exocytosis mechanisms in separate compartments and it is also common that more than one mechanism may coexist in the same compartment. For example, while the soma of leech serotonergic Retzius neurons releases serotonin exclusively from dense core vesicles (Trueta et al., 2003), synaptic clear vesicles in serotonergic neurons of leech or mammals are surrounded by serotonin-containing dense core vesicles that release their contents extrasynaptically (De-Miguel and Trueta, 2005). Electrical stimulation of serotonergic leech or mammalian neurons increases the serotonin concentration in the extracellular fluid microns away from the release sites, and serotonin release modulates the activity of neurons (Perrier and Cotel, 2008) and in addition inhibits the electrical activity of serotonergic neurons (Cercós et al., 2009). Increasing evidence shows that in response to extrasynaptic exocytosis, glial cells may take up and afterwards release transmitters, and by doing so may modulate neuronal activity.

Extrasynaptic transmission represents a subtype of volume (diffuse) transmission, namely the short-distance transmission of signaling molecules (inter alia transmitters, neuropeptides, and diffusible modulators) taking place in the extracellular fluid of local circuits (Fuxe and Agnati, 1991a,b; Descarries and Mechawar, 2000; Fuxe et al., 2007, 2010). Even the classical synaptic transmitter glutamate can be released to reach the extracellular fluid and activate extrasynaptic glutamate receptors located close to active glutamate synapses, as shown in brain slices, leading to increases in astroglial glutamate release (Del Arco et al., 2003). Glutamate as an astroglial derived volume transmission signal can then contribute to metabolic and trophic adjustments in the neuron-astrocytic unit in response to the activity of the glutamate synapses. Volume transmission also includes long distance diffusion in the extracellular fluid and the cerebrospinal fluid mainly mediated via neuropeptides like beta-endorphin and their fragments as well as proteins like, e.g., prolactin and Interleukin 1beta released from neurons and/ or from glial cells (Agnati et al., 1986).

This diversity of mechanisms and their effects adds complexity to the nervous system and raises many questions that still wait for answers. From the physiological point of view, one may ask how the neuronal firing pattern determines whether to evoke synaptic and/or extrasynaptic exocytosis in a given neuron; how activation of different synaptic inputs induces release from different release compartments, and by doing so affect different targets (Velazquez-Ulloa et al., 2003). From the behavioral point of view it becomes highly interesting to explore how the timing of circuits and therefore behaviors are modulated by extrasynaptic transmission. Some psychiatric and neurological dysfunctions, such as depression may be related to deficiencies in extrasynaptic transmission (Fuxe et al., 1991). A new research field is exploring whether antidepressant drugs used to reduce the symptoms of depression and/or of post-traumatic stress syndrome exert their effects by increasing the extracellular fluid levels of transmitters 
such as serotonin and thus volume transmission and by doing that restore the activity of distinct 5-HT receptor subtypes (Jansson et al., 2002), and in parallel activate neurogenesis, thus favoring mood elevation on one hand and the recovery of damaged brain areas on the other. This may occur at least in part through activating neurogenesis. Developmental and evolutionary biologists may also find the topic inspiring for future research, since some of the exocytosis mechanisms involved in extrasynaptic transmission evolved before chemical synapses. For a similar reason, one may expect that during development, the extracellular levels of transmitters, modulators, and peptides may contribute to axonal pathfinding and electrogenesis before massive synaptogenesis takes place.

\section{REFERENCES}

Agnati, L. F., Fuxe, K., Zoli, M., Ozini, I., Toffano, G., and Ferraguti, F. (1986). A correlation analysis of the regional distribution of central enkephalin and beta-endorphin immunoreactive terminals and of opiate receptors in adult and old male rats. Evidence for the existence of two main types of communication in the central nervous system: the volume transmission and the wiring transmission. Acta Physiol. Scand. 128, 201-207.

Agnati, L. F., Guidolin, D., Guescini, M., Genedani, S., and Fuxe, K. (2010). Understanding wiring and volume transmission. Brain Res. Rev. 64, 137-159.

Cercós, M., De-Miguel, F. F., and Trueta, C. (2009). Real-time measurements of synaptic autoinhibition produced by serotonin release in cultured leech neurons. J. Neurophysiol. 102, 1075-1085.

De-Miguel, F. F., and Trueta, C. (2005). Synaptic and extrasynaptic secretion of serotonin. Cell. Mol. Neurobiol. 25, 297-312.

Del Arco, A., Segovia, G., Fuxe, K., and Mora, F. (2003). Changes in dialysate concentrations of glutamate and GABA in the brain: an index of volume transmission mediated actions? J. Neurochem. 85, 23-33.

Descarries, L., and Mechawar, N. (2000). Ultrastructural evidence for diffuse transmission by monoamine and acetylcholine neurons of the central nervous system. Prog. Brain Res. 125, 27-47.
Fuxe, K., and Agnati, L. F. (eds). (1991b). Volume Transmission in the Brain: Novel Mechanisms for Neural Transmission. New York: Raven Press.

Fuxe, K., and Agnati, L. F. (1991a). "Wo principle modes of electrochemical communication in the brain: volume versus wiring transmission," in Volume Transmission in the Brain: Novel Mechanisms of Neuronal Transmission, eds K. Fuxe and L. F. Agnati (New York:

Fuxe, K., Dahlstrom, A., Hoistad, M., Marcellino, D., Jansson, A., Rivera, A., Diaz-Cabiale, Z., Jacobsen, K. Tinner-Staines, B., Hagman, B., Leo, G., Staines, W., Guidolin, D., Kehr, J., Genedani, S., Belluardo, N., and Agnati, L. F. (2007). From the GolgiCajal mapping to the transmitterbased characterization of the neuronal networks leading to two modes of brain communication: wiring and volume transmission. Brain Res. Rev. $55,17-54$.

Fuxe, K., Dahlström, A. B., Jonsson, G., Marcellino, D., Guescini, M., Dam, M., Manger, P., and Agnati, L. (2010). The discovery of central monoamine neurons gave volume transmission to the wired brain. Prog. Neurobiol. 90, 82-100.

Fuxe, K., Hedlund, P., von Euler, G., Lundgren, K., Martire, M., Ögren, S. O., Eneroth, P., and Agnati, L. F. (1991). "Galanin/5-HT interactions in the rat central nervous system. Relevance for depression," in Galanin. A New Multifunctional Peptide in the Neuroendocrine System, Wenner-Gren Int Series Vol 58, eds T. Hökfelt, T. Raven Press), 1-9.

Extrasynaptic transmission expands our view about how the nervous system works and also imposes new challenges to the way we plan our research. New technological developments are focused on imaging transmitters (Kaushalya et al., 2008a,b) and new computational tools are being developed to analyze transmitter mobilizations or long-term changes of neuronal circuit activity. New definitions and mechanisms may become visible. Meanwhile, this is a good moment for a first common effort to analyze and discuss extrasynaptic transmission in different systems and from different perspectives. We hope the readers of this issue will find the articles included of significant interest and helpful clarifying the field of extrasynaptic transmission.

Bartfai, D. Jacobowitz, and D. Ottoson (London: Macmillan Press), 221-235

Jansson, A., Descarries, L., CorneaHebert, V., Riad, M., Verge, D., Bancila, M., Agnati, L. F., Fuxe, K. (2002). "Transmitter-receptor mismatches in central dopamine serotonin and neuropeptide systems," in The Neurona Environment: Brain Homeostasis in Health and Disease, ed. W. Walz (Totowa, NJ: Humana Press), 83-107. Jansson, A., Lippoldt, A., Mazel, T., Bartfai, T., Ogren, S. O., Sykova, E., Agnati, L. F., and Fuxe, K. (2000). Long distance signalling in volume transmission. Focus on clearance mechanisms. Prog. Brain Res. 125, 399-413.

Joca, S. R., Guimarães, F. S., and Del-Bel, E. (2007). Inhibition of nitric oxide synthase increases synaptophysin mRNA expression in the hippocampal formation of rats. Neurosci. Lett. 421, 72-76.

Kaushalya, S. K., Nag, S., Ghosh, H., Arumugam, S., and Maiti, S. (2008a). A high-resolution large area serotonin map of a live rat brain section. Neuroreport 19, 717-721.

Kaushalya, S. K., Desai, R., Arumugam, S., Ghosh, H., Balaji, J., and Maiti, S. (2008b). Three-photon microscopy shows that somatic release can be a quantitatively significant component of serotonergic neurotransmission in the mammalian brain. J. Neurosci. Res. 86, 3469-3480.

Perrier, J. F., and Cotel, F. (2008). Serotonin differentially modulates the intrinsic properties of spinal motoneurons from the adult turtle. J. Physiol. (Lond.) 586, 1233-1238.
Trueta, C., Mendez, B., and De-Miguel, F. F. (2003). Somatic exocytosis of serotonin mediated by L-type calcium channels in cultured leech neurones. J. Physiol. (Lond.) 547, 405-416.

Trueta, C., Sanchez-Armass, S., Morales, M. A., and De-Miguel, F. F. (2004). Calcium-induced calcium release contributes to somatic secretion of serotonin in leech Retzius neurons. J. Neurobiol. 61, 309-316.

Velazquez-Ulloa, N., Blackshaw, S. E., Szczupak, L., Trueta, C., Garcia, E., and De-Miguel, F. F. (2003). Convergence of mechanosensory inputs onto neuromodulatory serotonergic neurons in the leech. J. Neurobiol. 54, 604-617.

Yuan, S., and Burrell, B. D. (2010). Endocannabinoid-dependent LTD in a nociceptive synapse requires activation of a presynaptic TRPV-like receptor. J. Neurophysiol. 104, 2766-2777.

Received: 22 December 2011; accepted: 24 January 2012; published online: 15 February 2012.

Citation: De-Miguel FF and Fuxe K (2012) Extrasynaptic neurotransmission as a way of modulating neuronal functions. Front. Physio. 3:16. doi: 10.3389/fphys.2012.00016 This article was submitted to Frontiers in Membrane Physiology and Biophysics, a specialty of Frontiers in Physiology. Copyright (C) 2012 De-Miguel and Fuxe. This is an open-access article distributed under the terms of the Creative Commons Attribution Non Commercial License, which permits non-commercial use, distribution, and reproduction in other forums, provided the original authors and source are credited. 